\title{
A Scoping Review Examining the Integration of Exercise Services in Clinical Oncology Settings
}

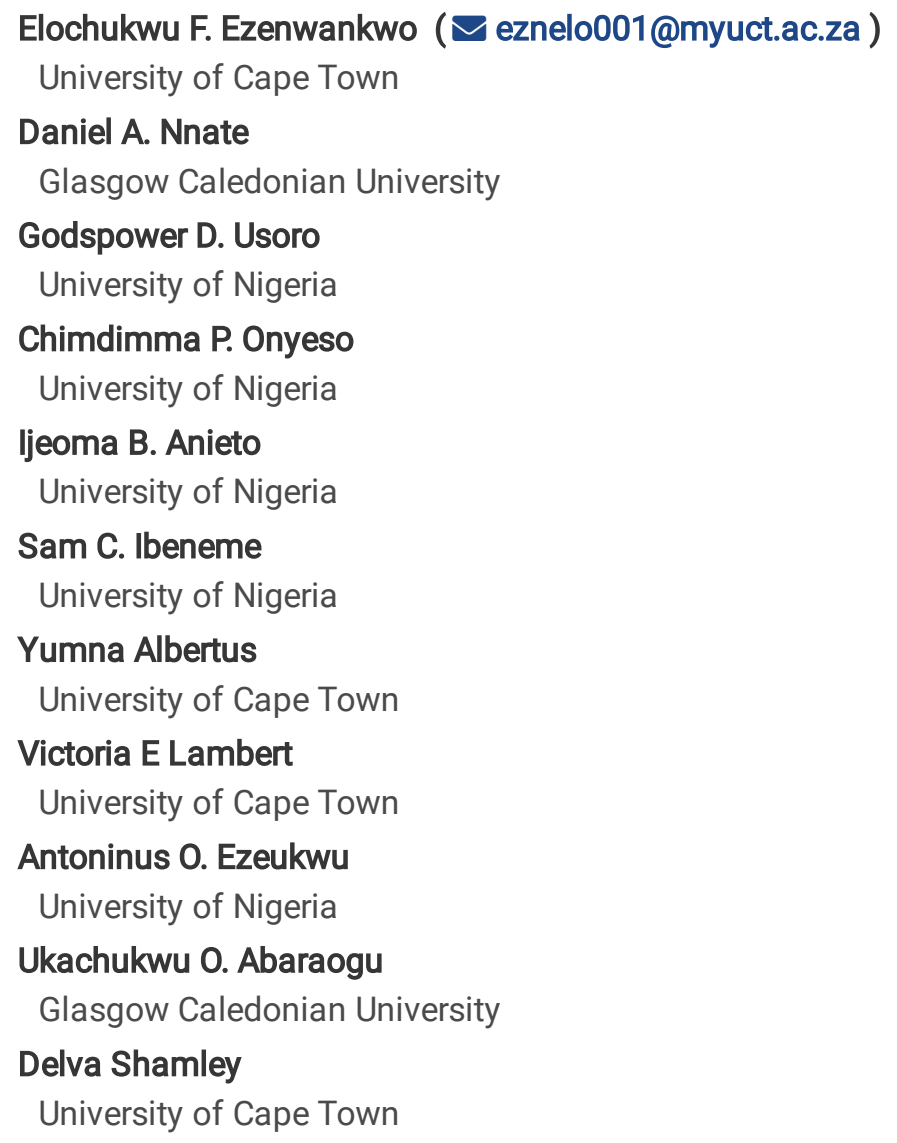

\section{Research Article}

Keywords: exercise services, service integration, reach, adoption, cost, utilization

Posted Date: September 14th, 2021

DOI: https://doi.org/10.21203/rs.3.rs-870829/v1

License: (c) (i) This work is licensed under a Creative Commons Attribution 4.0 International License. Read Full License

Version of Record: A version of this preprint was published at BMC Health Services Research on February 21st, 2022. See the published version at https://doi.org/10.1186/s12913-022-07598-y. 


\section{Abstract}

Background: Addressing questions surrounding the feasibility of embedding exercise service units in clinical oncology settings is imperative for developing a sustainable exercise-oncology clinical pathway. We examined available literature and provided practical recommendations to inform evidence-based practice, policymaking, and further investigations.

Methods: Studies that recruited cancer patients, assessed the co-location of exercise services and cancer treatment units and reported findings on service implementation were included. Evidence from six studies providing data from over 30 programs was integrated using narrative synthesis.

Results: Exercise service implementation was relatively modest across the included studies. These services were delivered by physiotherapists, exercise physiologists, and kinesiologists and funded mainly through grants and private donations, with staff salaries accruing as the major expense. Service penetration, adoption, and acceptability were generally low. However, studies recorded high clinician/patient satisfaction. Major barriers to service integration were limited funding, lack of detailed implementation plan, and low organizational buy-in. Common reasons for non-utilization, missed sessions, and dropouts were lack of interest, unwellness, hospital readmission, disease progression, and adverse skeletal events.

Conclusion: Implementing exercise services in clinical oncology settings seems an effective approach for increasing access to exercise medicine for individuals on cancer treatment. While this model appears feasible for patients/clinicians, calculated efforts are required to drive uptake. There is no one-size-fits-all approach; hence, sustainable service integration remains a product of many factors, including structures and strategies that reflect the organizational dynamics of the clinical service environment housing the exercise unit.

\section{Introduction}

The increasing global cancer prevalence and mortality raise deep concerns over the comparative magnitude of the disease impact on the global burden from chronic, non-communicable diseases (1). In 2020, more than 18 million new cancer cases were reported in 185 countries, with over 9 million associated deaths (2). From the point of diagnosis and throughout the balance of life, individuals with cancer are bound to experience several adverse outcomes with far-reaching consequences on their general wellbeing $(3,4)$. Regardless of cancer type and treatment regimen, most patients will experience chronic inflammation, fatigue and weakness, pain, sleep issues, altered body weight, gastrointestinal issues (i.e., bowel alterations, nausea, vomiting, etc.), psychosocial issues (i.e., anxiety, depression, fear, etc.), neurological issues (i.e., impaired cognition, poor coordination, and balance, etc.), respiratory issues (i.e., dyspnea, cough, etc.), oral and swallowing issues, loss of bladder and bowel control, erectile and sexual dysfunctions, skin issues (i.e., hot flashes, constant sweating, hair loss/appearance, etc.), musculoskeletal issues (i.e., frailty, osteoporosis, increased risk of falling and fracture) and edema $(3,5,6)$. These sequelae stem not only from the disease itself but considerably too from the multisystemic impacts of cancer treatment (7). These complications can persist in most patients for many years, even after treatments $(8,9)$. Many patients are bound to suffer both economic and social losses as a result: patients may experience loss of jobs, loss of [family] income, huge medical debt, social isolation, and ultimately the inability to participate in daily routine, vocational roles, and civic responsibilities (10-13).

From in vitro models, early preclinical studies and large population-based observational studies to high-quality clinical exercise efficacy trials and behavior change studies involving 'real world' scenarios, the cancer exercise literature abounds with clear and profound evidence of the mitigating effects and health benefits of exercise in the trajectory of cancer care (14-20) Research not only has shown that exercise is safe and feasible for cancer patients but also engaging in regular exercise from the point of diagnosis can improve treatment tolerance $(7,21,22)$, facilitate early recovery $(7,21,22)$, and reduce the length of hospital stay $(23,24)$. There is also evidence that exercise can slow cancer progression $(25)$, lower the risk of recurrence, readmission and mortality $(25,26)$. An improved exercise lifestyle also promises better quality of life $(7,27)$, early return to work and other day-today activities (7). Recent reports suggest that only about $30-47 \%$ of cancer patients are meeting current global exercise recommendations $(28,29)$. While many factors preclude cancer patients from engaging in regular exercise, lack of access to exercise medicine as part of routine care in oncology settings has remained a major barrier $(7,30)$. 
The call to make exercise medicine an integral component of routine oncology care is rapidly gaining traction globally as the World Health Organisation moves to increase global access to high-quality rehabilitation as essential healthcare service for individuals with chronic disease (7). Remarkably, many stakeholders are increasingly acknowledging a critical ramification of this 'call to action' to include embedding exercise services in cancer treatment settings (26). Oncology care models that foster integrative exercise-cancer care units may provide a more pragmatic approach for delivering access to timely, flexible, and highquality exercise medicine to cancer patients. When patients are offered early access to individualized and supervised exercise medicine, they are well-positioned to develop the physical, mental and psychosocial capacities to confront the challenges associated with cancer treatment even before they set in. Providing access to exercise-based rehabilitation within a cancer care setting is likely to encourage integrated and multidisciplinary oversight, creating opportunities for routine joint patient evaluation, shared decision making, and triage. A key benefit of this approach is that oncology clinicians, including doctors, nurses, and accredited exercise specialists, can recognize any potential risks/threats and intervene more holistically and timeously. This approach is bound to increase patients' confidence and satisfaction in their care. As exercise adoption and maintenance are particularly challenging in posttreatment populations, an integrated exercise-oncology care model may be the greatest leverage available to healthcare providers to intervene most critically within the window of time when patients are more amenable to behavior change (31).

Embedding an exercise service unit in a typical cancer treatment setting may present some challenges to patients, clinicians, and the health service system. First, the actual process of installing an exercise unit within existing treatment settings may require slight to huge (infra)structural (re)adjustments. The likely potential for disruption in workflow could come at a risk to patients as they may not be able to access routine care more efficiently during such time. Second, many health systems are currently grappling with underfunding globally. Hence even where integrative exercise-oncology models are less resource-intensive, health services may find it challenging to hire exercise specialists with the right credentials and experience for handling the peculiar exercise needs and challenges of cancer patients. Another critical factor is the capacity for patient screening, triage, and referral. As this is a relatively new frontier, the present clinical oncology workforce may lack the clarity, culture, and the will to assess, advise, and rightly refer patients for exercise medicine (26). Many facilities lack robust guideline-concordant care with well-defined and streamlined patient screening/evaluation algorithms and referral pathways (21). To effectively bridge this current evidencepractice gap, it is, therefore, imperative to begin by addressing questions around the feasibility, including the cost implications of implementing an exercise service unit in a standard oncology clinical setting to inform both evidence-based and cost-effective decisions.

\section{Methods}

\section{Research objective}

We aim to provide a comprehensive summary of peer-reviewed literature on the feasibility of implementing an exercise service unit within a cancer treatment setting. To achieve this, we performed a scoping review of the literature using the modified framework of Levac and colleagues (32). The current review does not warrant consent to participate or institutional ethics approval as only publicly available peer-reviewed literature were utilized, with no primary data collection (32). However, we reported our findings using the Preferred Reporting Items for Systematic reviews and Meta-Analyses extension for Scoping Reviews (PRISMA-ScR) guidelines (33).

\section{Study eligibility}

We included studies that evaluated the implementation of exercise service units within a cancer care setting in this review. To be eligible, service units were to have a well-defined structure and be located in a clinical setting (e.g., inpatient or outpatient services, public and private practice). Essentially, studies must report data for one of the following implementation outcomes: acceptability, adoption, appropriateness, practicality (including cost), feasibility, fidelity, penetration, sustainability, and quality assessment. As such, feasibility and efficacy trials evaluating exercise benefits in cancer population were excluded. We also included studies providing stakeholder perspectives on the co-location of exercise service and cancer care units. No restrictions were placed on study design or publication date. We also excluded non-primary research, including reviews, commentaries, and viewpoint articles, including non-English studies. 


\section{Information sources and search}

A comprehensive literature search was conducted on Embase via Ovid, CINAHL via EBSCOhost, MEDLINE via Ovid, Web of Science Core Collection via Clarivate Analytics and ProQuest (Health and Medicine) by EE and DN independently. EE and DN developed the search strategy using a well-defined systematic approach.(34) Relevant search terms and medical subject headings (MeSH) were identified by exploring the National Library of Medicine Database and, further, by reviewing a recent review of exercise intervention for cancer survivors (35). Specific keywords and MeSH terms applied in the search include (but are not limited to) cancer, exercise, feasibility, etc., and implementation outcomes such as acceptability, adoption, appropriateness, practicality (See Additional File 1). Additionally, recent systematic and meta-analytic reviews of cancer exercise literature were scanned for relevant citations.

\section{Article screening and selection}

Identified records were exported to RefWorks software for de-duplication and then Microsoft Excel Spreadsheet for screening. EE and DN independently screened the titles and abstracts of all retrieved citations and, further, the full texts of the remaining articles using the review's eligibility criteria. Differences in opinions at different points in the screening process were resolved by discussion in consultation with GU.

\section{Data extraction and analysis}

A data extraction form was developed and tested to guide the data extraction. First, we reviewed a variety of constructs as considered in the Implementation Outcome Framework of Proctor and Colleagues (36), Bowen's framework (37), and the Reach, Effectiveness, Adoption, Implementation, and Maintenance (RE-AIM) framework of Glasgow and colleagues (38), Next, we adapted a list of priority outcomes drawing on recent evidence and our experience in implementation research (Table 1). Data on study characteristics, cancer care setting, nature/components of exercise services, and implementation outcomes were extracted. This review focused on the following key implementation outcomes: implementation, cost, reach/penetration, service uptake/adoption, acceptability, patient satisfaction, fidelity, and sustainability. Quantitative and qualitative results were extracted, analyzed, and integrated to produce the final synthesis. 
Table 1

Operationalization of implementation outcomes

\begin{tabular}{|c|c|c|}
\hline Outcomes & Definition & Measurement metrics \\
\hline Reach/Penetration & $\begin{array}{l}\text { The absolute representativeness of individuals, including } \\
\text { healthcare providers and patients and organizations who are } \\
\text { willing to utilize exercise services integrated as part of cancer care }\end{array}$ & $\begin{array}{l}\text { - Total number of referrals to } \\
\text { exercise services relative to total } \\
\text { eligible patient population }\end{array}$ \\
\hline $\begin{array}{l}\text { Service uptake/ } \\
\text { adoption }\end{array}$ & $\begin{array}{l}\text { Service utilization by an organization as evidenced by reports on } \\
\text { the total number of staff referring patients to exercise service }\end{array}$ & - Number of patient referrers \\
\hline \multirow[t]{2}{*}{ Acceptability } & \multirow{2}{*}{$\begin{array}{l}\text { The number of patients who are willing to accept exercise } \\
\text { services and comply with exercise recommendations relative to } \\
\text { the total eligible patients }\end{array}$} & - Number of accepted referrals \\
\hline & & $\begin{array}{l}\text { - Service compliance (including } \\
\text { attrition) }\end{array}$ \\
\hline $\begin{array}{l}\text { Patient } \\
\text { satisfaction }\end{array}$ & $\begin{array}{l}\text { The extent to which exercise service is deemed suitable, } \\
\text { satisfactory and attractive to the patients }\end{array}$ & $\begin{array}{l}\text { - Any reports on patient } \\
\text { satisfaction including sources of } \\
\text { dissatisfaction }\end{array}$ \\
\hline \multirow[t]{5}{*}{ Implementation } & \multirow{5}{*}{$\begin{array}{l}\text { The extent to which exercise services are delivered to the intended } \\
\text { population successfully }\end{array}$} & - Workforce \\
\hline & & - Service operation \\
\hline & & - Access \\
\hline & & - Service promotion \\
\hline & & - Referral mechanism/pathway \\
\hline \multirow[t]{4}{*}{ Cost } & \multirow[t]{4}{*}{ The cost implications of service implementation } & - Funding source \\
\hline & & - Salaries \\
\hline & & - Purchase cost \\
\hline & & - Delivery cost \\
\hline Fidelity & $\begin{array}{l}\text { The degree of service providers' compliance with existing pre- } \\
\text { implementation plan, service protocols or standard operating } \\
\text { procedures }\end{array}$ & $\begin{array}{l}\text { - Documented efforts including } \\
\text { strategies to ensure fidelity } \\
\text { including consistency of service } \\
\text { delivery }\end{array}$ \\
\hline \multirow[t]{3}{*}{ Sustainability } & \multirow{3}{*}{$\begin{array}{l}\text { The extent to which exercise service becomes institutionalized as } \\
\text { standard in routine cancer care }\end{array}$} & - [infra]structural adjustments \\
\hline & & - Increased workforce \\
\hline & & - Increased funding \\
\hline
\end{tabular}

\section{Results}

\section{Study description}

Six studies providing data from over 30 exercise programs were included in this review (39-44). Details of the screening and selection process are provided in Fig. 1. One study $(43)$ was conducted in Canada, while the rest $(39-42,44)$ was carried out in Australia. Included studies were largely prospective involving varying cancer types and patient demographics except for Dennett et al., (40) a qualitative report on clinicians' perspectives. Patients were generally above 50 years and on active treatment with either chemotherapy, radiation therapy, immunotherapy. Of the included oncology services, two were publicly funded hospitals $(39,43)$, one was privately funded $(41,42)$, and one involved both public and private hospitals/cancer centers (44). Exercise services were individualized and group-based and largely featured a combination of aerobic and resistance exercise $(39,41-44)$. More details on the included studies are provided in Table 2. 
Table 2

Description of included studies

\begin{tabular}{|c|c|c|c|c|c|c|}
\hline $\begin{array}{l}\text { Author } \\
\text { Country }\end{array}$ & Study & Population & $\begin{array}{l}\text { Healthcare } \\
\text { setting }\end{array}$ & $\begin{array}{l}\text { Exercise } \\
\text { service }\end{array}$ & Service description & $\begin{array}{l}\text { Implementation } \\
\text { outcomes }\end{array}$ \\
\hline $\begin{array}{l}\text { Dennett } \\
2021 \\
(39) ; \\
2021 \\
(40) \\
\text { Australia }\end{array}$ & $\begin{array}{l}\text { Design: } \\
\text { Prospective } \\
\text { pre-post } \\
\text { design } \\
\text { Qualitative } \\
\text { evaluation } \\
\text { Evaluation: } 6 \\
\text { months }\end{array}$ & $\begin{array}{l}\text { Adult cancer } \\
\text { survivors ( } \mathrm{n}= \\
64 \text { ) currently } \\
\text { receiving or } \\
\text { preparing for } \\
\text { cancer treatment } \\
\text { (curative or } \\
\text { palliative) } \\
\text { admitted as an } \\
\text { inpatient or } \\
\text { outpatient } \\
\text { Age: } 63 \pm 11 y r s \\
\text { Gender: Male: } \mathrm{n} \\
=41 \text { (56\%); } \\
\text { Female: } \mathrm{n}=32 \\
\text { (44\%) } \\
\text { Physiotherapists } \\
\text { who delivered } \\
\text { the exercise } \\
\text { program and } \\
\text { referring } \\
\text { clinicians } \\
\text { (oncologists, } \\
\text { nurses, } \\
\text { physiotherapists, } \\
\text { occupational } \\
\text { therapists, social } \\
\text { workers) } \\
\text { working within } \\
\text { the cancer unit. }\end{array}$ & $\begin{array}{l}\text { Cancer unit - } \\
\text { inpatient } \\
\text { oncology ward } \\
\text { and outpatient } \\
\text { day oncology } \\
\text { center offering } \\
\text { chemotherapy - } \\
\text { embedded in a } \\
\text { publicly funded } \\
\text { tertiary hospital }\end{array}$ & $\begin{array}{l}\text { Exercise- } \\
\text { based } \\
\text { rehabilitation } \\
\text { within a } \\
\text { hospital- } \\
\text { based } \\
\text { cancer } \\
\text { treatment } \\
\text { center. }\end{array}$ & $\begin{array}{l}\text { Individually tailored, } \\
\text { Physiotherapist-led } \\
\text { group-based circuit } \\
\text { exercise class } \\
\text { Frequency: } 1-2 \\
\text { times/wk. } \\
\text { Duration: } 8 \text { weeks }\end{array}$ & $\begin{array}{l}\text { All } \\
\text { Clinician } \\
\text { perspective }\end{array}$ \\
\hline
\end{tabular}




\begin{tabular}{|c|c|c|c|c|c|c|}
\hline $\begin{array}{l}\text { Author } \\
\text { Country }\end{array}$ & Study & Population & $\begin{array}{l}\text { Healthcare } \\
\text { setting }\end{array}$ & $\begin{array}{l}\text { Exercise } \\
\text { service }\end{array}$ & Service description & $\begin{array}{l}\text { Implementation } \\
\text { outcomes }\end{array}$ \\
\hline $\begin{array}{l}\text { Kennedy } \\
2020 \\
(41) ; \\
\text { Newton } \\
2020 \\
(42) \\
\text { Australia }\end{array}$ & $\begin{array}{l}\text { Design: } \\
\text { Retrospective } \\
\text { evaluation } \\
\text { Evaluation: } \\
50 \text { months }\end{array}$ & $\begin{array}{l}\text { Individuals ( } \mathrm{n}= \\
73 \text { ) receiving } \\
\text { radiation therapy } \\
\text { and/or } \\
\text { chemotherapy } \\
\text { for different } \\
\text { cancer diagnosis } \\
\text { Median age: } \\
\text { 58.5 (IQR: 48- } \\
67) \\
\text { Gender: male: } \mathrm{n} \\
=21(32.8 \%) \\
\text { Female: } \mathrm{n}=43 \\
67.2 \%) ; \text { Cancer } \\
\text { type: Breast: } \mathrm{n}= \\
39,60.9 \% ; \\
\text { Prostate: } \mathrm{n}=13 \text {, } \\
20.3 \% ; \\
\text { Colorectal: } \mathrm{n}=3 \text {, } \\
4.7 \% ; \text { Lung: } \mathrm{n}=3 \\
\text { (4.7\%); Other: } \mathrm{n} \\
=6(9.4 \%) \\
\text { Secondary } \\
\text { cancer report: } \mathrm{n} \\
=3(4.8 \%) \\
\text { Treatment type: } \\
\text { Radiotherapy: } \mathrm{n} \\
=55(85.9 \%) ; \\
\text { Chemotherapy: } \\
\mathrm{n}=4(6.3 \%) ; \\
\text { Radiotherapy } \\
\text { and } \\
\text { chemotherapy: } \\
\mathrm{n}=5(7.8 \%)\end{array}$ & $\begin{array}{l}\text { Private oncology } \\
\text { care clinic } \\
\text { (GenesisCare) } \\
\text { providing } \\
\text { primarily } \\
\text { outpatient-based } \\
\text { radiation } \\
\text { therapy and } \\
\text { medical } \\
\text { oncology } \\
\text { treatments }\end{array}$ & $\begin{array}{l}\text { Exercise } \\
\text { service Clinic } \\
\text { (Co-LEC) } \\
\text { established } \\
\text { in } 2013 \text { by } \\
\text { researchers } \\
\text { from Edit } \\
\text { Cowan } \\
\text { University, in } \\
\text { partnership } \\
\text { with } \\
\text { GenesisCare }\end{array}$ & $\begin{array}{l}\text { Patient tailored } \\
\text { (progressive)/group- } \\
\text { based resistance } \\
\text { ( } 2-3 \text { sets; } 6-12 \\
\text { reps) + aerobic } \\
\text { exercise }(20 \text { mins; } \\
60 \%-80 \% \text { estimated } \\
\text { HRmax) delivered } \\
\text { by an AEP } \\
\text { Frequency: } \\
60 \text { mins/session; } 2- \\
3 \text { times/wk } \\
\text { Duration: } \\
\text { Throughout } \\
\text { treatment course } \\
\text { (Average: } 13 w \mathrm{ks})\end{array}$ & All \\
\hline
\end{tabular}




\begin{tabular}{|c|c|c|c|c|c|c|}
\hline $\begin{array}{l}\text { Author } \\
\text { Country }\end{array}$ & Study & Population & $\begin{array}{l}\text { Healthcare } \\
\text { setting }\end{array}$ & $\begin{array}{l}\text { Exercise } \\
\text { service }\end{array}$ & Service description & $\begin{array}{l}\text { Implementation } \\
\text { outcomes }\end{array}$ \\
\hline $\begin{array}{l}\text { Dalzell } \\
2017 \\
(43) \\
\text { Canada }\end{array}$ & $\begin{array}{l}\text { Design: } \\
\text { Prospective } \\
\text { Evaluation: } \\
60 \text { months }\end{array}$ & $\begin{array}{l}234 \text { patients: A } \\
\text { two-month } \\
\text { sample } \\
\text { evaluation } \\
\text { representing } 75 \\
\text { new and } 159 \\
\text { follow-up } \\
\text { patients with } \\
\text { multiple cancer } \\
\text { types. } \\
\text { Mean Age: } 52 \pm \\
15.5 y r s \\
\text { Female: } 65 \% \\
\text { Patients on } \\
\text { active treatment: } \\
52 \% \\
\text { Patients with } \\
\text { advanced } \\
\text { disease or } \\
\text { metastatic } \\
\text { cancer: } 35.5 \% \\
\text { Bone } \\
\text { metastasis: } 16 \%\end{array}$ & $\begin{array}{l}\text { Integrated } \\
\text { oncology and } \\
\text { palliative care } \\
\text { center within a } \\
\text { publicly funded } \\
\text { general hospital }\end{array}$ & $\begin{array}{l}\text { Multimodal } \\
\text { rehabilitation } \\
\text { care model } \\
\text { with } \\
\text { hospital- } \\
\text { based } \\
\text { exercise } \\
\text { oncology } \\
\text { referral } \\
\text { component } \\
\text { (ActivOnco) } \\
\text { embedded in } \\
\text { a cancer } \\
\text { center }\end{array}$ & $\begin{array}{l}\text { Individualized plus } \\
\text { group-based } \\
\text { multicomponent } \\
\text { exercise with patient } \\
\text { education, exercise } \\
\text { counseling, and } \\
\text { self-management }\end{array}$ & $\begin{array}{l}\text { All but } \\
\text { sustainability } \\
\text { and cost }\end{array}$ \\
\hline & & $\begin{array}{l}\text { Bone } \\
\text { metastasis: } 16 \%\end{array}$ & & & & \\
\hline $\begin{array}{l}\text { Dennett } \\
2017 \\
(44) \\
\text { Australia }\end{array}$ & $\begin{array}{l}\text { Design: Ex } \\
\text { post facto } \\
\text { design using } \\
\text { mixed } \\
\text { methods } \\
\text { approach } \\
\text { Evaluation: } 2 \\
\text { wks. }\end{array}$ & $\begin{array}{l}\text { Patients with } \\
\text { different cancer } \\
\text { diagnoses, } \\
\text { disease stages, } \\
\text { and treatment } \\
\text { status }\end{array}$ & $\begin{array}{l}\text { Public and } \\
\text { private } \\
\text { hospitals/cancer } \\
\text { centers across } 6 \\
\text { states/ } \\
\text { territories }\end{array}$ & $\begin{array}{l}31 \text { eligible } \\
\text { programs } \\
\text { identified } \\
\text { from } 56 \\
\text { public } \\
\text { settings and } \\
9 \text { private } \\
\text { settings }\end{array}$ & $\begin{array}{l}\text { Individualized } \\
\text { exercise program } \\
\text { (Block = } 14 \\
\text { programs; rolling = } \\
17 \text { programs) } \\
\text { comprising mainly a } \\
\text { combination of } \\
\text { aerobic, resistance, } \\
\text { and flexibility } \\
\text { exercise } \\
6-10 \\
\text { patients/session } \\
\text { Frequency/duration: } \\
\text { Outpatient: } 2 \\
\text { times/wk for } 8 \text { wks; } \\
\text { inpatient: } 2 \\
\text { times/day for the } \\
\text { duration of inpatient } \\
\text { stay (equivalent to } 2 \\
\text { wks) }\end{array}$ & $\begin{array}{l}\text { All but } \\
\text { sustainability } \\
\text { and cost } \\
\text { Clinician } \\
\text { perspectives }\end{array}$ \\
\hline
\end{tabular}

\section{Summary of implementation}

A summary of the implementation outcomes is provided in Table 3. 
Table 3

Summary of implementation outcomes

\begin{tabular}{|c|c|c|c|c|}
\hline & Dennett 2021,(39) 2021(40) & $\begin{array}{l}\text { Kennedy 2020,(41) Newton } \\
2020(42)\end{array}$ & Dalzell 2017(43) & Dennett 2017(44) \\
\hline Implementation & 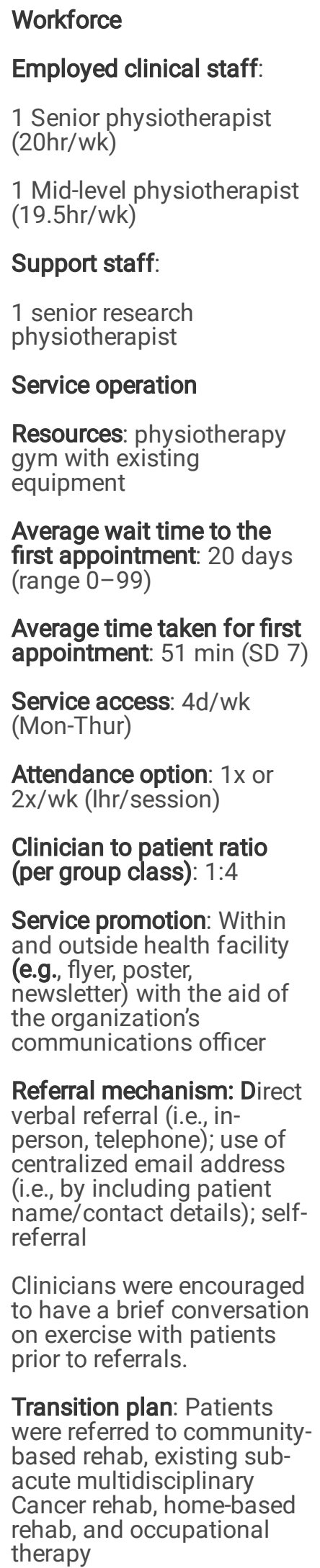 & $\begin{array}{l}\text { Workforce: } 4 \text { AEPs including } \\
\text { consultants (AEPs were } \\
\text { separate to the patient core } \\
\text { care team) } \\
\text { Service operation: } \\
\text { Independent of the cancer } \\
\text { center: patient triage and } \\
\text { integrated medical record } \\
\text { were lacking } \\
\text { Resources: Provided by ECU } \\
\text { Service access: 3days/wk; } \\
\text { 2hrs/session with lack of } \\
\text { co-ordination between gym } \\
\text { and treatment times } \\
\text { Service promotion: Not } \\
\text { reported } \\
\text { Referral mechanism } \\
\text { Pathway: direct verbal } \\
\text { referrals from clinicians; } \\
\text { self-referral } \\
\text { Referrals were made only } \\
\text { when oncologists } \\
\text { remembered and had the } \\
\text { time }\end{array}$ & $\begin{array}{l}\text { Workforce: } 5 \\
\text { physiotherapists (I } \\
\text { clinical director and } \\
4 \text { staff } \\
\text { physiotherapists) + } \\
3 \text { kinesiologists } \\
\text { with training and } \\
\text { experience in } \\
\text { oncology } \\
\text { Service operation: } \\
\text { Independent of the } \\
\text { cancer center } \\
\text { Resources: } \\
\text { Provided by Hope } \\
\text { and Care } \\
\text { Service promotion: } \\
\text { Presentations on } \\
\text { the value of } \\
\text { exercise } \\
\text { interventions to } \\
\text { various } \\
\text { departments } \\
\text { Referral } \\
\text { mechanism: well- } \\
\text { defined patient } \\
\text { triage and referral } \\
\text { pathways } \\
\text { Sources include } \\
\text { oncologists, allied } \\
\text { health workers, } \\
\text { self-referral, other } \\
\text { sources including } \\
\text { wellness centers } \\
\text { Transition plan: } \\
\text { Home-based } \\
\text { exercise program, } \\
\text { wellness center } \\
\text { the }\end{array}$ & $\begin{array}{l}\text { Workforce: } \\
\text { Physiotherapy: } \\
\text { 21/31 programs; } \\
\text { Exercise } \\
\text { Physiology: } \\
\text { 20/31 programs } \\
\text { Service } \\
\text { promotion: } \\
\text { Exercise fliers, } \\
\text { letters to GPs, } \\
\text { community } \\
\text { awareness } \\
\text { programs } \\
\text { Service structure: } \\
\text { outpatient } \\
\text { programs: } 2 x / \text { wk } \\
\text { for } 8 \text { wks; } \\
\text { inpatient } \\
\text { programs: } \\
\text { 2x/day for the } \\
\text { duration of } \\
\text { inpatient stay } \\
\text { (approximately } 2 \\
\text { weeks) } \\
\text { Early morning } \\
\text { sessions were } \\
\text { less practical and } \\
\text { received the } \\
\text { lowest patient } \\
\text { attendance } \\
\text { Developing } \\
\text { flexible and } \\
\text { rolling program is } \\
\text { critical to } \\
\text { enhance } \\
\text { practicality } \\
\text { Referral } \\
\text { mechanism } \\
\text { Patient feedback } \\
\text { to their primary } \\
\text { doctors was a } \\
\text { key driver of } \\
\text { more referrals } \\
\text { from doctors } \\
\text { Transition plan: } \\
\text { gome-based } \\
\text { groups }\end{array}$ \\
\hline
\end{tabular}




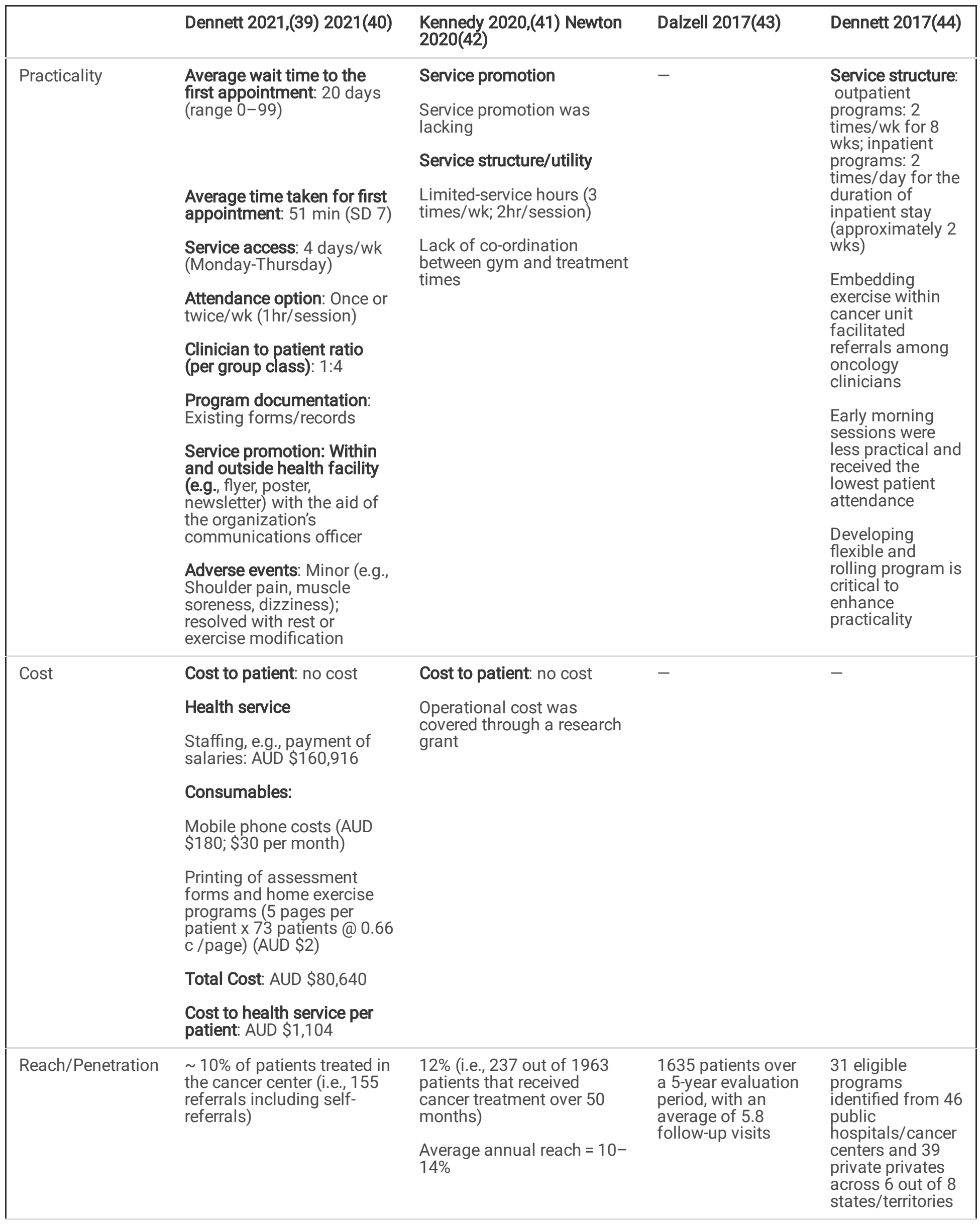




\begin{tabular}{|c|c|c|c|c|}
\hline & Dennett 2021,(39) 2021(40) & $\begin{array}{l}\text { Kennedy 2020,(41) Newton } \\
\text { 2020(42) }\end{array}$ & Dalzell 2017(43) & Dennett 2017(44) \\
\hline Service uptake & $\begin{array}{l}46 \text { staff made } 148 \text { referrals } \\
\text { over the } 6 \text { months } \\
\text { evaluation period: medical: } \\
n=32 \text { ( } 22 \%) \text {; nurses: } n=53 \\
(36 \%) \text {; allied health: } n=63 \\
(4 \%) \\
\text { Facilitators: Service } \\
\text { visibility, convenience, } \\
\text { building rapport, } \\
\text { accessibility, timing, and } \\
\text { staff experience }\end{array}$ & $\begin{array}{l}\text { Sources of referrals: } \\
\text { oncologists = } 21 \% \text {; nurses = } \\
20 \% \\
\text { Number of oncologists with } \\
\text { at least } 1 \text { patient attending } \\
\text { Co-LEC }=11 / 11\end{array}$ & $\begin{array}{l}\text { Referral sources } \\
\text { over a 2-month } \\
\text { period: Oncologists } \\
=35 \% \text {; allied health: } \\
36 \%\end{array}$ & $\begin{array}{l}\text { Referral sources: } \\
\text { oncologists } \\
\text { ( } 28 / 31 \\
\text { programs); allied } \\
\text { health clinicians } \\
\text { ( } 21 / 31 \\
\text { programs) } \\
\text { Barrier: Low } \\
\text { exercise literacy } \\
\text { among doctors }\end{array}$ \\
\hline Acceptability & $\begin{array}{l}34 \%(52 * \text { out of eligible } 199 \\
\text { patients) } \\
\text { Refused referrals: } n=67 \\
(43 \%), \text { reasons: not } \\
\text { interested }(n=17), \text { unsure } \\
(n=16), \text { unwell/treatment } \\
\text { related }(n=3) \text {, work }(n=2) \text {, } \\
\text { location/parking }(n=2), \\
\text { home-based exercise }(n= \\
21) \text { other ( }=6) \\
\text { No. of refusals after } 1 \text { st } \\
\text { session: } n=2 \text { (reason: } \\
\text { readmission = } 1) \\
\text { Compliance: } 38 \text { patients } \\
\text { elected for } 2 \text { times/wk with } \\
56 \% \text { completing } 7 / 16 \\
\text { sessions; } 14 \text { patients } \\
\text { elected for } 1 \text { times/wk with } \\
40 \% \text { completing } 3 / 8 \\
\text { sessions } \\
\text { Missed sessions were due } \\
\text { to: } \\
\text { Refusal (25\%) } \\
\text { Unwell due to treatment } \\
\text { (23\%) } \\
\text { Drop out: } n=20 ; 38 \% \\
\text { (Reasons: CoViD- } 19 \\
\text { restrictions; hospital } \\
\text { readmission, disease } \\
\text { progression) }\end{array}$ & $\begin{array}{l}27 \% \text { (i.e., } 64 \text { out of } 237 \\
\text { referrals over a } 50 \text { month) } \\
\text { Common reason for non- } \\
\text { service utilization was lack } \\
\text { of awareness of its } \\
\text { availability }\end{array}$ & $\begin{array}{l}71 \% \text { compliance } \\
\text { (over a } 3 \text { year) in a } \\
\text { sample of } 41 \\
\text { patients with } \\
\text { multiple myeloma } \\
\text { ( } 81 \% \text { had bone } \\
\text { lesion) on active } \\
\text { treatment } \\
\text { Dropouts: } \\
\text { Increased with the } \\
\text { incidence of } \\
\text { skeletal-related } \\
\text { events, including } \\
\text { pathologic fracture, } \\
\text { spinal cord } \\
\text { compression, and } \\
\text { radiation for } \\
\text { stabilization of } \\
\text { bone lesions }\end{array}$ & $\begin{array}{l}\text { Overall, annual } \\
\text { enrolment per } \\
\text { program: } 10-70 \\
\text { patients } \\
2000 \text { survivors } \\
\text { per year across } \\
\text { Australia }\end{array}$ \\
\hline
\end{tabular}




\begin{tabular}{|c|c|c|c|c|}
\hline & Dennett 2021,(39) 2021(40) & $\begin{array}{l}\text { Kennedy 2020,(41) Newton } \\
2020(42)\end{array}$ & Dalzell 2017(43) & Dennett 2017(44) \\
\hline Satisfaction & $\begin{array}{l}\mathrm{n}=57(100 \%) \\
\text { Access (timing, facility, } \\
\text { location): } \mathrm{n}=46 \text { (81\%) } \\
\text { Willingness to recommend } \\
\text { others to participate during } \\
\text { treatment: } \mathrm{n}=57 \text { (100\%) } \\
\text { Feeling of improved overall } \\
\text { health/wellbeing: } \mathrm{n}=56 \\
(98 \%) \\
\text { Sources of dissatisfaction } \\
\text { Difficulties with access: } \mathrm{n}= \\
6 \text { ( } 8 \%) \\
\text { Difficulties were largely due } \\
\text { to lack of parking space }\end{array}$ & $\begin{array}{l}\text { Social value: } n=11 \text { out of } \\
61 \text { patients } \\
\text { Improved treatment } \\
\text { experience: } 12 \text { out of } 61 \\
\text { patients } \\
\text { Positivity: } 24 / 61 \text { patients: } \\
\text { Staff } \\
\text { experience/professionalism: } \\
17 / 61 \text { patients } \\
\text { Sources of dissatisfaction } \\
\text { Lack of coordination } \\
\text { between treatment and gym } \\
\text { times: } 33 / 51 \text { patients } \\
\text { Parking issues: } 5 / 51 \\
\text { patients } \\
\text { Lack of transition plan at } \\
\text { the end of the program: } \\
4 / 51 \text { patients }\end{array}$ & - & $\begin{array}{l}\text { Patient cantered: } \\
\text { programs } \\
\text { addressed } \\
\text { individual patient } \\
\text { needs and goals } \\
\text { Programs } \\
\text { increased } \\
\text { opportunities for } \\
\text { social support } \\
\text { Sources of } \\
\text { dissatisfaction } \\
\text { program timing } \\
\text { (attendance were } \\
\text { lowest for early } \\
\text { morning } \\
\text { sessions) } \\
\\
\text { Parking issues } \\
\text { travel distances } \\
\text { particularly for } \\
\text { metropolitan } \\
\text { centers }\end{array}$ \\
\hline Fidelity proxy & $\begin{array}{l}\text { Exercise service was } \\
\text { implemented by clinicians } \\
\text { with } 5.5 \text { years oncology- } \\
\text { specific experience and } \\
\text { prior cancer-specific } \\
\text { training in acute and } \\
\text { community cancer settings. } \\
\text { A steering committee } \\
\text { comprising a consumer, } \\
\text { clinical directors, } \\
\text { physiotherapy manager } \\
\text { and a community partner } \\
\text { ensured service } \\
\text { implementation } \\
\text { Program staff and other } \\
\text { hospital physiotherapists } \\
\text { received three } 1 \text { hr } \\
\text { education sessions on } \\
\text { cancer and rehabilitation } \\
\text { Medical, nursing, and allied } \\
\text { health staff received } 3 \\
\text { presentations to provide } \\
\text { updates throughout } \\
\text { program implementation }\end{array}$ & $\begin{array}{l}\text { Service implementation was } \\
\text { spearheaded by } 3 \text { AEPs with } \\
\text { experience in exercise } \\
\text { oncology }\end{array}$ & $\begin{array}{l}\text { Continuous staff } \\
\text { mentoring and } \\
\text { education }\end{array}$ & - \\
\hline
\end{tabular}




\begin{tabular}{|c|c|c|c|c|}
\hline & Dennett 2021,(39) 2021(40) & $\begin{array}{l}\text { Kennedy 2020,(41) Newton } \\
\text { 2020(42) }\end{array}$ & Dalzell 2017(43) & Dennett 2017(44) \\
\hline \multirow[t]{8}{*}{ Sustainability } & $\begin{array}{l}\text { Philanthropic funds were } \\
\text { sought to pay staff salaries } \\
\text { to sustain the program } \\
\text { beyond the pilot period }\end{array}$ & $\begin{array}{l}\text { Funding: Direct clinical } \\
\text { operational cost was } \\
\text { covered by ECU and } \\
\text { GenesisCare to support } \\
\text { service continuation at the } \\
\text { end of the feasibility phase }\end{array}$ & - & - \\
\hline & & $\begin{array}{l}\text { Structural adjustments } \\
\text { (mainly due to inadequate } \\
\text { funds): }\end{array}$ & & \\
\hline & & $\begin{array}{l}\text { Operational hours reduced } \\
\text { from } 6 \mathrm{hrs} / \mathrm{wk} \\
(2 \mathrm{hrs} / \text { days/wk) to } 2 \mathrm{hrs} / \mathrm{wk} \\
(1 \mathrm{hr} / 2 \text { days/wk) }\end{array}$ & & \\
\hline & & $\begin{array}{l}\text { Eligibility was rescinded for } \\
\text { patients receiving } \\
\text { chemotherapy alone }\end{array}$ & & \\
\hline & & $\begin{array}{l}\text { Service duration was } \\
\text { reduced to } 3 \text { months for all } \\
\text { patients regardless of } \\
\text { treatment duration }\end{array}$ & & \\
\hline & & Challenges & & \\
\hline & & $\begin{array}{l}\text { Communication gap } \\
\text { between ECU and } \\
\text { GenesisCare }\end{array}$ & & \\
\hline & & $\begin{array}{l}\text { Financial model was } \\
\text { lacking- Co-LEC was not } \\
\text { generating revenue }\end{array}$ & & \\
\hline
\end{tabular}

\section{Service implementation}

Exercise services were largely operated independently of the housing treatment settings, and program staff was generally not part of the patient core care team $(39,41,43,44)$. Exercise services were delivered by physiotherapists $(39,43,44)$, exercise physiologists $(41,44)$, and kinesiologists (43) experienced in oncology settings. One study reported a clinician-to-patient ratio of 1:4 (39). Access to exercise services varied across the included studies. In one study, exercise sessions were available every Monday to Thursday, and participants have access to a one-hour gym session once or twice a week (39). In another study, participants were provided access to three exercise sessions per week, with each session lasting two hours.(41). In Dennett et al., (44) outpatients accessed programs twice a week for eight weeks while inpatients attended up to two sessions per day for the entire duration of their hospital stay. Early morning sessions and lack of coordination between treatment and gym times were reported as key barriers to program access $(41,44)$. Structured patient referral mechanism was generally lacking except for one study that showed evidence of a well-designed patient triage and referral pathways (43). Referrals were largely verbal, from the oncologist and other healthcare providers directly to the exercise programs $(39,41,44)$. Self-referrals were also reported in all the included studies $(39,41,43,44)$. One study reported using an email system to create a central access point for the clinicians (39). Exercise programs were promoted differently across the included studies. Strategies such as flyers $(39,44)$, posters (39), newsletters (39), letters to general practitioners (44), community awareness (44), and in-hospital presentations (43) were adopted to promote the programs within and outside the health facilities. At program completion, patients were largely recommended for home-based exercise programs $(39,43,44)$, a hospital-based multidisciplinary rehabilitation program (39) or community-based rehabilitation programs $(39,43,44)$. 
Exercise programs were delivered at no cost to the patients; however, operational costs were largely covered with public funds, including grants $(39,41)$ and private donations $(43)$. Staff salaries accrued a greater part of the operational cost $(39,41)$. In one study, the per-patient cost to the health service was AUD \$1,104 (41).

\section{Reach}

Program reach as reported in two studies was 10\% (39) and 12\% (41), with Kennedy et al. (41) reporting an annual reach of 10$14 \%$ over a 50 -month evaluation period. In one study,(43) 1635 patients were evaluated in 5 years with an average of 5.8 follow-up visits. Another study identified only 31 programs from 85 public and private hospitals/cancer centers in 6 out of 8 states/territories in Australia (44).

\section{Service uptake}

Individual referral data were generally lacking. In one study, 46 staff made 148 referrals over a 6-month evaluation period (39). In another study, all the oncologists $(n=11)$ consulting in the cancer treatment center had at least one patient under their management attending exercise clinic at any time within the 50-month evaluation period (41). Referrals were largely from doctors, nurses, and allied health staff $(39,41,43,44)$. Referrals from nurses were around $20 \%(41)$ and $36 \%(39,43)$ of the total referrals. Referrals from doctors were largely poor -i.e., $21-22 \%(39,41)$ and $35 \%(43)$. Factors that improved service uptake among clinicians were patient feedback, regular service promotion, enhanced visibility, convenience, building rapport, accessibility, timing, and staff experience $(39,40,44)$. Poor knowledge among doctors on the role of exercise in cancer management was reported as a major barrier to service uptake (44).

\section{Acceptability}

Two studies reported $27 \%$ (39) and $44 \%$ (41) acceptance rates. One study reported $71 \%$ compliance in a sample of 41 patients over three years (43). In one study, $56 \%$ of the participants elected for three weekly exercise sessions attended 7 out of 16 sessions (39). In the same study, $40 \%$ of the participants electing for once per week exercise sessions attended only 3 out of 8 sessions (39). One study reported 10-70\% annual enrolment per program ( $\mathrm{n}=31$ programs), averaging 2000 cancer survivors per year across Australia (44). Common reasons for non-utilization, missed sessions, and dropout were COVID-19 restrictions (39), hospital readmission (39), disease progression (39), lack of awareness of service availability (41), adverse skeletal events,(43) unwell due to treatment (39), and patient refusal (39).

\section{Patient satisfaction}

Patient satisfaction was high amid varying cancer types and patient demographics. In one study, $81 \%$ of the total responders ( $\mathrm{n}=$ 46) were satisfied with the facility, location, and timing of the program, and all the responders $(n=57)$ reported their willingness to refer other patients to participate in the program during treatment (39). Key drivers of patient satisfaction were improved wellbeing and overall treatment experience $(39,41)$, staff experience and professionalism $(41)$, social value $(39,41,44)$, feeling of empowerment (41), and patient-centered service (44). Program timing (44), lack of coordination between gym and treatment times (41) parking issues $(39,41,44)$, travel distance $(44)$, and lack of transition plan (41) were frequently mentioned as major causes of dissatisfaction.

\section{Fidelity proxy}

None of the studies provided reports on service fidelity. However, to ensure a high standard of care, service implementation was largely done by clinicians with experience in the oncology setting $(39,41,43,44)$. This was further ensured by a steering committee comprising a consumer, clinical directors, physiotherapy manager, and a community partner (39). Other approaches maintained to ensure a high-quality service delivery include regular updates(39) and continuous staff mentoring and education (43).

\section{Sustainability}


To sustain exercise services beyond the evaluation period, in one study, philanthropic funds were sought to pay staff salaries (39). In another study (41), besides the partnership between the exercise service providers and the cancer care center to cover operational costs, program duration was reduced to three months. Also, the operational hours were scaled down to two days per week (one hour per session) (41). Eligibility was further rescinded for patients receiving chemotherapy alone (41). In the same study, lack of a financial model and effective communication between the partnering organizations were the major threats to the program's sustainability (41).

\section{Discussion}

The impetus for the current review stems primarily from the growing need to close the research-practice gap that has long existed in the field of exercise oncology. Even with the overwhelming evidence on the feasibility, safety, and clinical benefits of exercise in cancer patients, exercise-based rehabilitation is still generally considered an adjunct instead of an integral component of care during treatment. The result of this evidence-practice gap is that most patients do not have access to exercise medicine while receiving cancer treatment, a period when the debilitating effects of cancer treatments are at their peak and can best be mitigated or ameliorated with exercise medicine $(21,26,45)$. Despite a limited number of studies, implementing exercise service in [proximity to] a cancer unit appears to be an effective approach for increasing access to exercise medicine for individuals on active treatment (39-44). While this approach seems to be feasible for both the clinicians (referring clinicians and those delivering the program) and patients, the current evidence is not a confirmation of the overall feasibility of exercise service integration in oncology care settings. The lack of a clear implementation plan was evident across the included studies (39-44). As critical to a successful service implementation as this may be, issues relating to funding and organizational buy-in hold even far greater implications for effective service integration and sustainability.

Overall, service implementation was modest even though fidelity to any pre-implementation plan, service protocols, or standard operating procedures were not demonstrated in any of the included studies. As a direct consequence of this downside, capacity for patient screening and risk stratification, effective patient triage, and structured referral mechanisms were mostly lacking. Exercise services were largely operated independently of the clinical settings they were embedded, and staff leading these programs were generally not part of the patient core management team $(41,44)$. This compromised the potential for shared decision-making in most programs and enabled communication gaps between the clinical staff and exercise service providers (41, 44). Lack of an implementation plan was implicated in the poor coordination between exercise sessions and treatment time. In one study, patients reported that they could not attend exercise sessions because they constantly clashed with their treatment times (41). As co-location does not automatically translate to successful service integration, a detailed implementation plan ensures that structures and strategies that reflect the changing dynamics of the clinical environment housing an exercise service unit are put in place to drive effective and sustainable integration.

Access to exercise programs were relatively feasible and similar across the included studies. Most programs were open to participants two to three times a week $(39,41,43,44)$. In one study, for example, exercise services were available from Monday to Thursday during the six months evaluation period (39). In another study, patients on admission had daily access to exercise programs throughout their inpatient stay (44). Another consistent finding across the included studies was the simplified and convenient nature of the referral process $(39,41,43,44)$. Although well-defined referral pathways were generally lacking, patient referrals were simple and convenient. Exercise referrals were mostly verbal, directly from the referring clinicians (the oncologists, nurses, and other allied health staff) to the exercise programs $(39,41,44)$. One study reported an additional use of a central access point (email referral) to facilitate patient referrals further.(39) Another major facilitator of referrals was patient feedback $(39,40,44)$. One study reported that doctors who received positive feedback on the exercise program directly from their patients were more inclined to refer more patients to the exercise program (40). Barriers to patient referrals were recorded at both individual and health service levels. At the individual level, while most doctors were aware of exercise benefits, particularly during cancer treatment, many lacked the will to refer patients to exercise programs. In one study, doctors reported referring patients to the exercise program only when they remembered and had the time to do so (41). At the health service level, low organizational buy-in, even with the reported evidence of adequate service promotion, was a major finding $(39,41,43,44)$.

Lack of organizational buy-in may be responsible for the overall low service penetration and utilization among the clinicians. For example, two studies reported overall service reach ranging between $10 \%$ and $12 \%(39,41)$, with Kennedy et al $(41)$. reporting an

Page 15/22 
annual reach of $10-14 \%$ over a 50 -month evaluation period. One study identified only 31 exercise service programs in the whole of 6 out of 8 states/territories in Australia (44). Successful integration of exercise services in routine oncology care demands a concerted effort to develop and identify the right implementation strategies to provoke a cultural shift in the host organization, which is critical for increasing organizational buy-in. One way to achieve this is by providing education to the healthcare providers working in oncology settings. Healthcare providers can only refer patients to exercise service programs if they know how, when, and where patients can be referred for such services (21). To refer patients for exercise services, doctors and nurses, for example, should understand and appreciate the rehabilitation dimensions of their patient care and effectively and proactively screen patients for exercise interventions $(21,45)$. As this is a relatively new frontier in cancer care, many healthcare providers in oncology settings may need to be trained on how to use exercise screening algorithms and referral guidelines to adopt these tools $(21,45)$. Tools such as electronic medical records and integrated/central referral systems can improve service ease and efficiency, and ultimately utilization $(21,45)$.

Organizational buy-in can also be improved by enhancing the visibility of exercise service units. In one study, referring clinicians were pleased with the value created by the frequent presence of physiotherapists in the cancer unit as they actively feature in ward rounds, offer clinical insights even during informal discussions, and took part in patient assessment and decision making (40). Another strategy to increase service utilization is to increase staff confidence, particularly in the safety of the exercise program (40). One way to achieve this is to ensure that the physiotherapists and other exercise specialists working in these settings have the right skills and training to match the peculiarities and dynamics of cancer care. Good communication, knowledge sharing, patient responsiveness, and teamwork can foster strong relationships between staff leading exercise programs and oncology clinicians and ultimately enhance service utilization (40). Regular service promotion within the clinical setting is another strategy to increase organizational buy-in. One study achieved this by providing regular updates and timely reminders through staff presentations, the use of newsletters, and by introducing an alerting system in electronic medical records $(39,40,43,44)$. Staff rotations and turnover reflect the dynamics of typical cancer care clinics. Regular awareness programs are thus critical to ensure that new staff is aware of the existence of these services.

The acceptance rate was relatively low across the included studies. One study, for example, found that only 64 patients took part in the exercise program out of 237 referrals received over 50 months (41). Another study reported that almost half of eligible patients referred to exercise programs declined their referrals (39). In one study, some programs recorded even as low as $10 \%$ annual enrolment (44). Service compliance was also low among those that participated in the exercise program except for one study that recorded as high as $71 \%$ compliance over a three-year evaluation period (43), One reason that could explain the reported low service acceptance is the poor referral process. While the referral process was found to be simple and convenient for the clinicians, it may have lacked some critical elements that guaranty an effective referral mechanism, one of which is patient engagement. To illustrate, common reasons for non-utilization, missed sessions, and dropouts across the included studies were lack of interest (39), unwell due to treatment (39), COVID-19 restrictions (39), hospital readmission (39), disease progression (39), lack of awareness of service availability (41), and worsening symptoms including adverse skeletal events (43). These experiences appear to be underlined by a general lack of exercise self-efficacy and behavioral control which is a common observation in patients on active cancer treatment $(46,47)$. The poor understanding of the complex nature of cancer disease and the appropriate exercise dose with minimal adverse effects required to derive health benefits are also potential accentuating factors among these patients $(46,47)$. The referral process offers the treating oncologists and nurses a unique opportunity to motivate the right attitudes and positive intentions, empowering their patients to build the ultimate conviction in their capabilities to initiate and maintain routine exercise behavior. Oversimplifying the referral process robs the referring clinicians of opportunities to engage proactively with and counsel patients prior to their exercise journey. The observed low service uptake further speaks to the peculiar challenges of patients on active cancer treatment, especially those on hospital admission. These individuals constantly battle with multiple treatment-related complications and are generally unwell. Offering regular counseling and psychosocial supports and adapting exercise programs to reflect individual capacities, needs, and preferences can be another useful approach to increase uptake. Even though most patients were satisfied and willing to refer others to these programs, low service uptake and high dropout rates can be improved especially in the outpatient population by addressing sources of dissatisfaction, such as program timing (i.e., by scheduling sessions early in the morning), lack of coordination between exercise and treatment times (i.e., by creating a more flexible programs), lack of continuation plans, and parking issues (i.e., by eliminating or subsidizing parking fees) $(39,41,44)$. 
Issues relating to funding also pose a major threat to sustainable service integration. Even though exercise services can be delivered with less sophisticated equipment, funds are required to cover routine operational costs, including daily consumables, staff salaries, maintenances, and in some locations, rents. Most of the programs were funded through grants and private donations $(39,41,43,44)$. These sources are largely volatile and unsustainable. In one study, the average cost of the exercise service per patient was AUD $\$ 1,104$ with staff salaries being the primary expense (41). Most of the programs could not be sustained after the evaluation period, largely due to inadequate resources. For example, one study reported that two programs were closed because of a lack of funds (44). In another study, the authors reported that the exercise program was restructured at the end of the evaluation period to ensure that the available funds are used to cover the basic operational costs (41). Funding is a key driver of long-term service and should form primary consideration during the program planning phase. As health systems continue to grapple with limited resource allocation globally, funding challenges are even more pronounced in exercise oncology, given the pervasive misconception about rehabilitation as largely an adjunctive service. Governments, corporate sponsors, and insurance agencies are potential opportunities that could be explored for multiple funding streams.(48) More research is therefore required to confirm the greater merits of integrative exercise-cancer care models to the broader health systems. This can provoke a cultural shift in healthcare funding policies to guaranty sustainable funding for exercise rehabilitation.

The strength of this review is evident in our reliance on multiple implementation outcome frameworks. By drawing extensively on well-established frameworks, our findings and recommendations are comprehensive and robust and can effectively guide clinical practice, policy formulation, and further investigations. One major limitation of the current review is the limited number of studies included. Further, as per the aim of our review, we did not evaluate the potential of this approach in translating into measurable clinical benefits. Again, by excluding non-English articles, we may have missed out on studies that could strengthen our findings and recommendations further.

\section{Conclusion}

Addressing questions around the feasibility of embedding an exercise service unit in clinical oncology settings is imperative for developing a sustainable exercise-oncology clinical pathway. While this appears to be an effective approach for increasing access to exercise-based rehabilitation for individuals on active cancer treatment, the current findings reveal major challenges with service penetration, adoption, and utilization. Issues relating to funding, lack of detailed implementation plan, and low organizational buy-in were the major barriers to effective service integration, particularly at the health service level. Common reasons for non-utilization, missed sessions, and dropouts were lack of interest, unwellness due to treatment, COVID-19 restrictions, hospital readmission, disease progression, lack of awareness of service availability, and adverse skeletal events. While this model appears feasible for clinicians and patients, calculated efforts are required to drive uptake. As there is no onesize-fits-all approach, sustainable service integration remains a product of many factors, including structures and strategies that reflect the organizational dynamics of the clinical service environment housing the exercise unit.

\section{List Of Abbreviations}

PRISMA

Preferred Reporting Items for Systematic Review and Meta-Analysis

CINAHL

Cumulative Index to Nursing and Allied Health Literature

\section{Declarations}

\section{Ethics approval and consent to participate}

This study is a scoping review of publicly available peer-reviewed literature, with no primary data collection. Hence, it does not warrant consent to participate or institutional review board approval.

Page $17 / 22$ 


\section{Consent for publication}

Not applicable

\section{Availability of data and materials}

All data generated and analysed during this study are included in this manuscript [and its supplementary information files].

\section{Competing interests}

The authors declare that they have no competing interests.

\section{Funding}

Not applicable

\section{Authors' contributions}

EE, DN YA, VL, and DS conceptualized the study; EE and DN developed the review protocol and drafted the initial manuscript; EE, DN, GU, CO and IA led data collection, analysis, and interpretation; SI, AE, UA, YA, VL, and DS provided critical insights and reviewed the final draft; all authors read and approved the final manuscript.

\section{Acknowledgments}

Not applicable.

\section{References}

1. Global health estimates: Leading causes of DALYs [Internet]. [cited 2021 Jul 5]. Available from: https://www.who.int/data/gho/data/themes/mortality-and-global-health-estimates/global-health-estimates-leading-causesof-dalys

2. Sung H, Ferlay J, Siegel RL, Laversanne M, Soerjomataram I, Jemal A, et al. Global Cancer Statistics 2020: GLOBOCAN Estimates of Incidence and Mortality Worldwide for 36 Cancers in 185 Countries. CA: A Cancer Journal for Clinicians [Internet]. 2021 May [cited 2021 Jul 5];71(3):209-49. Available from: https://pubmed.ncbi.nlm.nih.gov/33538338/

3. Henry DH, Viswanathan HN, Elkin EP, Traina S, Wade S, Cella D. Symptoms and treatment burden associated with cancer treatment: Results from a cross-sectional national survey in the U.S. Supportive Care in Cancer [Internet]. 2008 Jul [cited 2021 Jul 5];16(7):791-801. Available from: https://pubmed.ncbi.nlm.nih.gov/18204940/

4. Woźniak K, Izycki D. Cancer: A family at risk [Internet]. Vol. 13, Przeglad Menopauzalny. Termedia Publishing House Ltd.; 2014 [cited 2021 Jul 5]. p. 253-61. Available from: /pmc/articles/PMC4520372/

5. Reilly CM, Bruner DW, Mitchell SA, Minasian LM, Basch E, Dueck AC, et al. A literature synthesis of symptom prevalence and severity in persons receiving active cancer treatment. Supportive Care in Cancer [Internet]. 2013 Jun [cited 2021 Jul 5];21(6):1525-50. Available from: /pmc/articles/PMC4299699/

6. Brearley SG, Stamataki Z, Addington-Hall J, Foster C, Hodges L, Jarrett N, et al. The physical and practical problems experienced by cancer survivors: A rapid review and synthesis of the literature. European Journal of Oncology Nursing [Internet]. 2011 Jul [cited 2021 Jul 5];15(3):204-12. Available from: https://pubmed.ncbi.nlm.nih.gov/21489873/

7. Stout NL, Santa Mina D, Lyons KD, Robb K, Silver JK. A systematic review of rehabilitation and exercise recommendations in oncology guidelines. CA: A Cancer Journal for Clinicians [Internet]. 2021 Mar 1 [cited 2021 Jul 5];71(2):149-75. Available from: https://acsjournals.onlinelibrary.wiley.com/doi/full/10.3322/caac.21639

8. Huang IC, Hudson MM, Robison LL, Krull KR. Differential impact of symptom prevalence and chronic conditions on quality of life in cancer survivors and non-cancer individuals: A population study. Cancer Epidemiology Biomarkers and Prevention [Internet]. 2017 Jul 1 [cited 2021 Jul 5];26(7):1124-32. Available from: https://pubmed.ncbi.nlm.nih.gov/28336581/ 
9. Schmidt ME, Wiskemann J, Steindorf K. Quality of life, problems, and needs of disease-free breast cancer survivors 5 years after diagnosis. Quality of Life Research [Internet]. 2018 Aug 1 [cited 2021 Jul 5];27(8):2077-86. Available from: https://pubmed.ncbi.nlm.nih.gov/29740782/

10. Paltrinieri S, Fugazzaro S, Bertozzi L, Bassi MC, Pellegrini M, Vicentini M, et al. Return to work in European Cancer survivors: a systematic review [Internet]. Vol. 26, Supportive Care in Cancer. Springer Verlag; 2018 [cited 2021 Jul 5]. p. 2983-94. Available from: https://pubmed.ncbi.nlm.nih.gov/29845421/

11. Lee YH, Goo-Yoshino S, Lew HL, Chi WC, Yen CF, Liao HF, et al. Social participation in head and neck cancer survivors with swallowing disorder: World Health Organization Disability Assessment Schedule 2.0 study. Head and Neck [Internet]. 2020 May 1 [cited 2021 Jul 5];42(5):905-12. Available from: https://pubmed.ncbi.nlm.nih.gov/31886608/

12. Carlotto A, Hogsett VL, Maiorini EM, Razulis JG, Sonis ST. The economic burden of toxicities associated with cancer treatment: Review of the literature and analysis of nausea and vomiting, diarrhoea, oral mucositis and fatigue [Internet]. Vol. 31, PharmacoEconomics. Pharmacoeconomics; 2013 [cited 2021 Jul 5]. p. 753-66. Available from: https://pubmed.ncbi.nlm.nih.gov/23963867/

13. Granström B, Tiblom Ehrsson Y, Holmberg E, Hammerlid E, Beran M, Tano K, et al. Return to work after oropharyngeal cancer treatment-Highlighting a growing working-age population. Head and Neck [Internet]. 2020 Aug 1 [cited 2021 Jul 5];42(8):1893-901. Available from: https://pubmed.ncbi.nlm.nih.gov/32092226/

14. Ezenwankwo EF, Ezeukwu AO, Abaraogu UO. Effects of physical activity changes induced by behaviour change interventions on inflammation and patient-centred outcomes in breast cancer survivors: a systematic review [Internet]. European Journal of Physiotherapy. Taylor and Francis Ltd.; 2021 [cited 2021 Jul 5]. Available from: https://www.tandfonline.com/doi/abs/10.1080/21679169.2021.1933586

15. Morishita S, Hamaue Y, Fukushima T, Tanaka T, Fu JB, Nakano J. Effect of Exercise on Mortality and Recurrence in Patients With Cancer: A Systematic Review and Meta-Analysis [Internet]. Vol. 19, Integrative Cancer Therapies. SAGE Publications Inc.; 2020 [cited 2021 Jul 5]. Available from: https://doi.org/10.1177/1534735420917462

16. Ashcraft KA, Peace RM, Betof AS, Dewhirst MW, Jones LW. Efficacy and Mechanisms of Aerobic Exercise on Cancer Initiation, Progression, and Metastasis: A Critical Systematic Review of In Vivo Preclinical Data [Internet]. Vol. 76, Cancer Research. American Association for Cancer Research Inc.; 2016 [cited 2021 Jul 5]. p. 4032-50. Available from: https://pubmed.ncbi.nlm.nih.gov/27381680/

17. Brown MJ, Morris MA, Akam EC. An exploration of the role of exercise in modulating breast cancer progression in vitro: a systematic review and meta-analysis [Internet]. Vol. 320, American Journal of Physiology - Cell Physiology. American Physiological Society; 2021 [cited 2021 Jul 5]. p. C253-63. Available from: https://journals.physiology.org/doi/abs/10.1152/ajpcell.00461.2020

18. Galvão DA, Spry N, Denham J, Taaffe DR, Cormie P, Joseph D, et al. A multicentre year-long randomised controlled trial of exercise training targeting physical functioning in men with prostate cancer previously treated with androgen suppression and radiation from TROG 03.04 radar. European Urology [Internet]. 2014 [cited 2021 Jul 5];65(5):856-64. Available from: https://pubmed.ncbi.nlm.nih.gov/24113319/

19. Lahart IM, Metsios GS, Nevill AM, Carmichael AR. Physical activity for women with breast cancer after adjuvant therapy [Internet]. Vol. 2018, Cochrane Database of Systematic Reviews. John Wiley and Sons Ltd; 2018 [cited 2021 Jul 5]. Available from: https://pubmed.ncbi.nlm.nih.gov/29376559/

20. Kenfield SA, Stampfer MJ, Giovannucci E, Chan JM. Physical activity and survival after prostate cancer diagnosis in the health professionals follow-up study. Journal of Clinical Oncology [Internet]. 2011 Feb 20 [cited 2021 Jul 5];29(6):726-32. Available from: https://pubmed.ncbi.nlm.nih.gov/21205749/

21. NL S, JC B, AL S, TF M, AM C, L N, et al. An exercise oncology clinical pathway: Screening and referral for personalized interventions. Cancer [Internet]. 2020 Jun 15 [cited 2021 Jul 7];126(12):2750-8. Available from: https://pubmed.ncbi.nlm.nih.gov/32212338/

22. Stout NL, Silver JK, Baima J, Knowlton SE, Hu X. Prehabilitation: An Emerging Standard in Exercise Oncology. Exercise Oncology [Internet]. 2020 [cited 2021 Jul 7];111-43. Available from: https://link.springer.com/chapter/10.1007/978-3-03042011-6_6 
23. Schmitz K, Campbell A, Stuiver M, Pinto B, Schwartz A, Morris G. Exercise is medicine in oncology: Engaging clinicians to help patients move through cancer. CA: A Cancer Journal for Clinicians. 2019;69(6):468-484.

24. Haiderali A, Menditto L, Good M, Teitelbaum A, Wegner J. Impact on daily functioning and indirect/direct costs associated with chemotherapy-induced nausea and vomiting (CINV) in a US population. Supportive Care in Cancer. 2011;19(6):843-851.

25. Campbell KL, Winters-Stone KM, Patel A V., Gerber LH, Matthews CE, May AM, et al. An executive summary of reports from an international multidisciplinary roundtable on exercise and cancer: Evidence, guidelines, and implementation. Rehabilitation Oncology [Internet]. 2019 Oct 1 [cited 2021 Jul 7];37(4):144-52. Available from: https://journals.Iww.com/rehabonc/Fulltext/2019/10000/An_Executive_Summary_of_Reports_From_an.3.aspx

26. Schmitz KH, Campbell AM, Stuiver MM, Pinto BM, Schwartz AL, Morris GS, et al. Exercise is medicine in oncology: Engaging clinicians to help patients move through cancer. CA: A Cancer Journal for Clinicians [Internet]. 2019 Nov 1 [cited 2021 Aug 3];69(6):468-84. Available from: https://acsjournals.onlinelibrary.wiley.com/doi/full/10.3322/caac.21579

27. Effinger KE, Stratton KL, Fisher PG, Ness KK, Krull KR, Oeffinger KC, et al. Long-term health and social function in adult survivors of paediatric astrocytoma: A report from the Childhood Cancer Survivor Study. European Journal of Cancer [Internet]. 2019 Jan 1 [cited 2021 Jul 5];106:171-80. Available from: https://pubmed.ncbi.nlm.nih.gov/30528801/

28. Galvão DA, Newton RU, Gardiner RA, Girgis A, Lepore SJ, Stiller A, et al. Compliance to exercise-oncology guidelines in prostate cancer survivors and associations with psychological distress, unmet supportive care needs, and quality of life. Psycho-Oncology [Internet]. 2015 Oct 1 [cited 2021 Jul 5];24(10):1241-9. Available from: https://onlinelibrary.wiley.com/doi/full/10.1002/pon.3882

29. Thraen-Borowski KM, Gennuso KP, Cadmus-Bertram L. Accelerometer-derived physical activity and sedentary time by cancer type in the United States. PLoS ONE [Internet]. 2017 Aug 1 [cited 2021 Jul 5];12(8). Available from: https://pubmed.ncbi.nlm.nih.gov/28806753/

30. Galvão DA, Chambers SK. Exercise medicine in men with prostate cancer: breaking barriers to increase participation [Internet]. Prostate Cancer and Prostatic Diseases. Springer Nature; 2021 [cited 2021 Jul 5]. p. 1-2. Available from: https://doi.org/10.1038/s41391-021-00406-4

31. Rabin C. Promoting Lifestyle Change Among Cancer Survivors: When Is the Teachable Moment?: http://dx.doi.org/101177/1559827609338148 [Internet]. 2009 Jun 11 [cited 2021 Aug 2];3(5):369-78. Available from: https://journals.sagepub.com/doi/abs/10.1177/1559827609338148

32. Levac D, Colquhoun H, O'Brien KK. Scoping studies: advancing the methodology. Implementation Science 2010 5:1 [Internet]. 2010 Sep 20 [cited 2021 Jul 9];5(1):1-9. Available from: https://implementationscience.biomedcentral.com/articles/10.1186/1748-5908-5-69

33. AC T, E L, W Z, KK O, H C, D L, et al. PRISMA Extension for Scoping Reviews (PRISMA-ScR): Checklist and Explanation. Annals of internal medicine [Internet]. 2018 Oct 2 [cited 2021 Jul 9];169(7):467-73. Available from: https://pubmed.ncbi.nlm.nih.gov/30178033/

34. Bramer WM, Jonge GB de, Rethlefsen ML, Mast F, Kleijnen J. A systematic approach to searching: an efficient and complete method to develop literature searches. Journal of the Medical Library Association: JMLA [Internet]. 2018 Oct 1 [cited 2021 Jul 9];106(4):531. Available from: /pmc/articles/PMC6148622/

35. Czosnek L, Richards J, Zopf E, Cormie P, Rosenbaum S, Rankin NM. Exercise interventions for people diagnosed with cancer: a systematic review of implementation outcomes. BMC Cancer 2021 21:1 [Internet]. 2021 May 30 [cited 2021 Jul 9];21(1):125. Available from: https://bmccancer.biomedcentral.com/articles/10.1186/s12885-021-08196-7

36. Proctor E, Silmere H, Raghavan R, Hovmand P, Aarons G, Bunger A, et al. Outcomes for Implementation Research: Conceptual Distinctions, Measurement Challenges, and Research Agenda. Administration and Policy in Mental Health and Mental Health Services Research 2010 38:2 [Internet]. 2010 Oct 19 [cited 2021 Jul 9];38(2):65-76. Available from: https://link.springer.com/article/10.1007/s10488-010-0319-7

37. Bowen DJ, Kreuter M, Spring B, Cofta-Woerpel L, Linnan L, Weiner D, et al. How We Design Feasibility Studies. American journal of preventive medicine [Internet]. 2009 May [cited 2021 Aug 10];36(5):452. Available from: /pmc/articles/PMC2859314/ 
38. Glasgow RE, Harden SM, Gaglio B, Rabin B, Smith ML, Porter GC, et al. RE-AIM Planning and Evaluation Framework: Adapting to New Science and Practice With a 20-Year Review. Frontiers in Public Health. 2019;0(MAR):64.

39. AM D, B Z, R W, SB T, K W, CL P. Bridging the gap: a pre-post feasibility study of embedding exercise therapy into a co-located cancer unit. Supportive care in cancer: official journal of the Multinational Association of Supportive Care in Cancer [Internet]. 2021 [cited 2021 Aug 16]; Available from: https://pubmed.ncbi.nlm.nih.gov/33963458/

40. Dennett AM, Peiris CL, Tan G, Shields N. Clinician's perspectives of implementing exercise-based rehabilitation in a cancer unit: a qualitative study. Supportive Care in Cancer 2021 [Internet]. 2021 Jul 5 [cited 2021 Aug 25];1-8. Available from: https://link.springer.com/article/10.1007/s00520-021-06378-4

41. Kennedy MA, Bayes S, Galvão DA, Singh F, Spry NA, Davis M, et al. If you build it, will they come? Evaluation of a co-located exercise clinic and cancer treatment centre using the RE-AIM framework. European Journal of Cancer Care. 2020 Jul 1;29(4).

42. Newton RU, Kennedy MA, Singh F, Taaffe DR, Spry NA, Chee R, et al. Safety, Effectiveness, and Uptake of Exercise Medicine Integrated Within a Cancer Care Center. Seminars in Oncology Nursing. 2020 Oct 1;36(5):151073.

43. Dalzell MA, Smirnow N, Sateren W, Sintharaphone A, Ibrahim M, Mastroianni L, et al. Rehabilitation and exercise oncology program: translating research into a model of care. Current Oncology [Internet]. 2017 [cited 2021 Aug 16];24(3):e191. Available from: /pmc/articles/PMC5486391/

44. AM D, CL P, N S, D M, NF T. Exercise therapy in oncology rehabilitation in Australia: A mixed-methods study. Asia-Pacific journal of clinical oncology [Internet]. 2017 Oct 1 [cited 2021 Aug 16];13(5):e515-27. Available from: https://pubmed.ncbi.nlm.nih.gov/28004526/

45. Schmitz KH, Stout NL, Maitin-Shepard M, Campbell A, Schwartz AL, Grimmett C, et al. Moving through cancer: Setting the agenda to make exercise standard in oncology practice. Cancer. 2021 Feb 1;127(3):476-84.

46. HEL E, CC F, C V, DA G, RU N, G W, et al. Examining the Priorities, Needs and Preferences of Men with Metastatic Prostate Cancer in Designing a Personalised eHealth Exercise Intervention. International journal of behavioral medicine [Internet]. 2021 Aug 1 [cited 2021 Aug 26];28(4):431-43. Available from: https://pubmed.ncbi.nlm.nih.gov/32968943/

47. G S, E G, LO N, D H, J H. The views of patients with metastatic prostate cancer towards physical activity: a qualitative exploration. Supportive care in cancer: official journal of the Multinational Association of Supportive Care in Cancer [Internet]. 2018 Jun 1 [cited 2021 Aug 26];26(6):1747-54. Available from: https://pubmed.ncbi.nlm.nih.gov/29243168/

48. Mina DS, Alibhai SMH, Matthew AG, Guglietti CL, Steele J, Trachtenberg J, et al. Exercise in clinical cancer care: a call to action and program development description. Current Oncology [Internet]. 2012 [cited 2021 Aug 26];19(3):e136. Available from: /pmc/articles/PMC3364774/

\section{Figures}



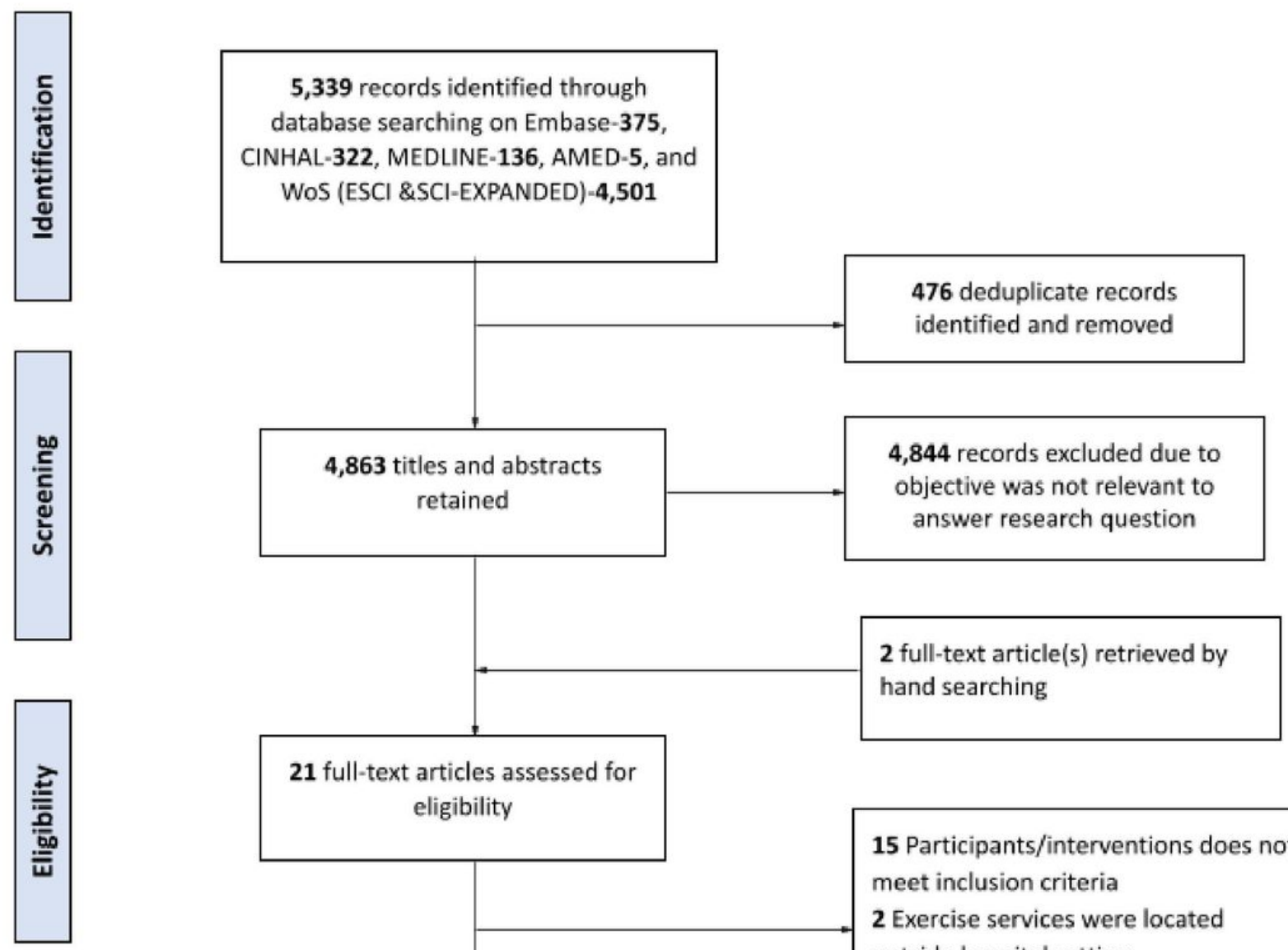

21 full-text articles assessed for eligibility

15 Participants/interventions does not meet inclusion criteria

2 Exercise services were located

outside hospital setting

1 Review paper

2 Conference paper

6 studies (prospective $=4$;

mixed methods $=1$;

qualitative $=1$ )

Figure 1

PRISMA flow diagram of the study selection procedure

\section{Supplementary Files}

This is a list of supplementary files associated with this preprint. Click to download.

- AdditionalFile1.docx 